\title{
Transmission Electron Microscopy Study on the Crystallization of Ion Beam Assisted Deposited CoFeB/MgO/CoFeB Magnetic Tunnel Junctions with Tantalum Capping Layer
}

\author{
R.V. Petrova ${ }^{1}$, R. Ferreira ${ }^{1,2}$, S. Cardoso ${ }^{2,3}$, P. P. Freitas ${ }^{2,3}$, S.McVitie ${ }^{4}$ and J.N. Chapman ${ }^{4}$ \\ ${ }^{1}$ International Iberian Nanotechnology Laboratory (INL), Braga, Portugal \\ ${ }^{2}$ Microsystems and Nanotechnology (INESC-MN), Lisbon, Portugal \\ ${ }^{3}$ Department of Physics, IST, Lisbon, Portugal \\ ${ }^{4}$ School of Physics and Astronomy, University of Glasgow, Glasgow, United Kingdom
}

Magnetic tunnel junctions (MTJs) have been studied extensively over the last ten years due to their ability to demonstrate a large tunneling magneto resistance ratio (TMR) at room temperature. MTJs have been applied to high-density magnetic recording heads and magnetic random access memory (MRAM), and recently MgO-based MTJs are replacing the aluminum oxide-based ones as a result of their higher TMR ratio. The $\mathrm{CoFeB} / \mathrm{MgO} / \mathrm{CoFeB}$ structure with its excellent crystal coherency [1] is the important factor for the high TMR value; hence the crystalline quality and texture of both $\mathrm{MgO}$ and $\mathrm{CoFeB}$ are of great significance for the resulting TMR.

Ion beam assisted deposition was proposed earlier [2] as an alternative to the more traditional PVD as a method to produce $\mathrm{MgO} / \mathrm{CoFeB}$ MTJs [3]. We use this approach starting from a ceramic $\mathrm{MgO}$ target, aiming the optimization of the assist beam conditions, in order to promote the crystallization of both $\mathrm{MgO}$ and the adjacent $\mathrm{CoFeB}$. XRD spectra of $30 \mathrm{~nm}$-thick $\mathrm{MgO}$ on glass show that $\mathrm{MgO}$ deposited without the assistance source is always amorphous. The crystallization of $\mathrm{MgO}$ can be promoted using the assist beam either with high power (concurrent etching regime) or low power (soft energy transfer regime). Stacks of $\mathrm{Ta}(3) /[\mathrm{CoFeB}(2) / \mathrm{MgO}(3)] \mathrm{xN} / \mathrm{CoFeB}(2) / \mathrm{Ta}(3)[\mathrm{nm}]$ were deposited on glass substrates using either regime for the $\mathrm{MgO}$ growth and annealed at $360^{\circ} \mathrm{C}$ for 1 hour.

Cross-sectional transmission electron microscopy (TEM) specimens were prepared using focused ion beam (FEI Nova 200 DualBeam) in situ lift-out technique and examined in a field emission TEM/STEM (FEI Tecnai TF 20) equipped with Gatan Enfina electron energy loss spectroscopy (EELS) spectrometer. Conventional bright (BF) and dark field (DF), high resolution (HR) TEM and high-angle annular dark field (HAADF) STEM imaging, EELS spectrum imaging and selected area diffraction (SAD) were used to obtain structural and chemical information from the layers in the MTJ stacks.

Lattice fringes in the HRTEM images (FIG. 1) indicate that both growth conditions provide crystalline $\mathrm{MgO}$ with (001) texture and, upon annealing, CoFeB layers with (001) preferred orientation. It is found that, no matter which deposition regime is used, the $\mathrm{CoFeB}$ layers crystallize in the bcc structure upon annealing and always have good (001) texture as they grow on $\mathrm{MgO}$ with strong (001) texture. Lower magnification DF images (FIG. 2) acquired with (020) reflection for fcc $\mathrm{MgO}$ and (200) reflection for bcc $\mathrm{CoFeB}$ support this finding although there are some (110) oriented crystallites in the $\mathrm{CoFeB}$ layers for both growth conditions.

EELS spectrum imaging was used to study the elemental distribution in all layers and it was determined that boron was hardly present in the stack deposited under the concurrent etching regime. Significant amount of boron existed in the $\mathrm{CoFeB} / \mathrm{Ta}$ interface deposited using the soft energy transfer regime. These results suggest that $\mathrm{CoFeB}$ crystallizes at the $\mathrm{MgO} / \mathrm{CoFeB}$ interface and 
boron diffuses from that interface to the Ta capping layer; Ta layer thus blocks the diffusion of boron.

\section{References}

[1] Y. S. Choi et al., J.Appl. Phys., vol. 101 (2007) 013907.

[2] S. Cardoso et al., J. Appl. Phys. Vol 103 (April 2008) 07A905-07907.

[3] Butler et al., Phys.Rev. B 63 (2001) 056614.

[4] The assistance of Dr. Sam McFadzean and Dr. Ian MacLaren from the University of Glasgow is gratefully acknowledged.

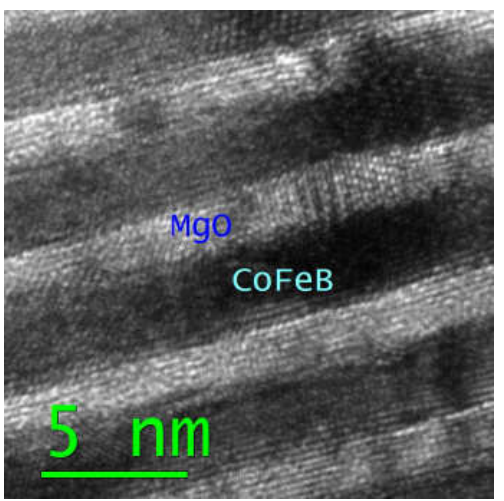

(a)

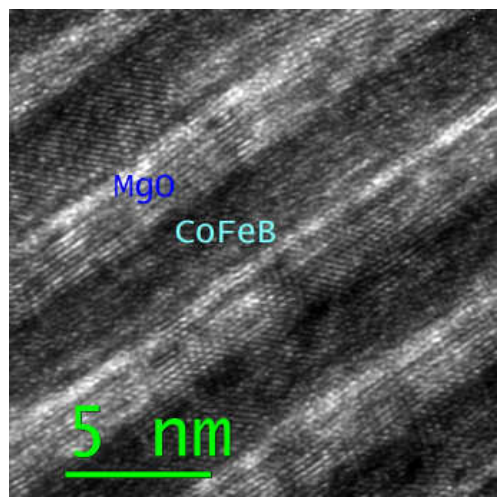

(b)

FIG.1. HRTEM images show lattice fringes in all layers of the stacks deposited using: (a) the concurrent etching and (b) the soft energy transfer regime.

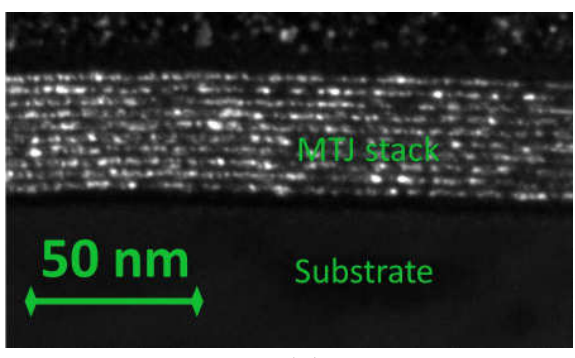

(a)

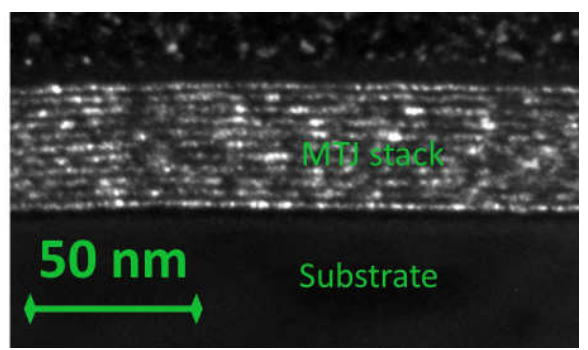

(b)

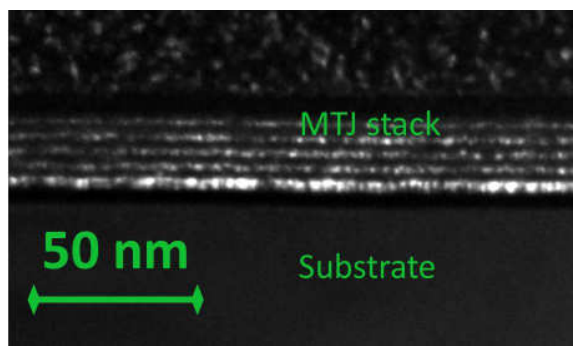

(c)

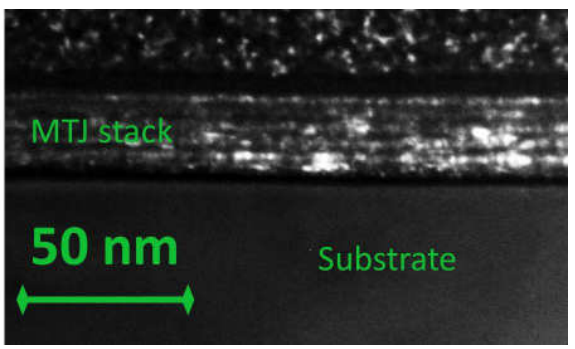

(d)

FIG.2. DF TEM images of the stack cross-sections show preferred (001) orientation in $\mathrm{MgO}$ and CoFeB layers. Stacks in: (a), (b) were deposited in high power regime and in (c), (d) using low power regime. DF images were taken: (a), (c) with (020) $\mathrm{MgO}$; (b), (d) with (200) $\mathrm{CoFeB}$ reflections. 